\title{
Intoxicação por Cestrum intermedium (Solanaceae) em bovinos no Estado de Santa Catarina
}

\author{
Cestrum intermedium (Solanaceae) poisoning in cattle of the Santa Catarina State
}
Fernando Henrique Furlan ${ }^{1}$, J oelma Lucioli ${ }^{1}$, Vanessa Borelli ${ }^{1}$, Oscar Olívio de Faria J unior ${ }^{2}$, Sandro Volnei Rebelatto ${ }^{2}$, Aldo Gava ${ }^{1} \&$ Sandra Davi Traverso ${ }^{1}$

\begin{abstract}
RESUMO
Intoxicação por plantas de ação hepatotóxica aguda constantemente é causa de mortes em animais de produção, principalmente em bovinos. Cestrum intermedium é um arbusto da família Solanaceae que ocorre principalmente no extremo oeste de Santa Catarina, sudoeste do Paraná e noroeste do Rio Grande do Sul. É conhecido popularmente como coerana e mataboi. No extremo oeste de Santa Catarina é a planta tóxica de maior importância para bovinos. Descreve-se um surto de intoxicação espontânea por Cestrum intermedium em bovinos no município de Jupiá, oeste de Santa Catarina. O quadro clínico era caracterizado por agressividade, incoordenação, fasciculação muscular, decúbito esternal, evoluindo para decúbito lateral seguido de movimentos de pedalagem e morte. As principais lesões macroscópicas foram observadas no fígado e consistiam em evidenciação do padrão lobular. Na histologia, observou-se necrose hepática centrolobular à massiva, acompanhada de congestão centro lobular e degeneração vacuolar dos hepatócitos adjacentes. Nenhuma outra planta hepatotóxica aguda foi encontrada no local onde os bovinos permaneciam.
\end{abstract}

Descritores: Cestrum intermedium, intoxicação, bovinos.

\section{ABSTRACT}

Poisoning by plants that cause liver necrosis constantly results in death in livestock, mainly in cattle. Cestrum intermedium is a bush of family Solanaceae that occurs mainly in the western of Santa Catarina, southwestern of Paraná and northwestern of Rio Grande do Sul. Is popularly known as coreana and mata-boi. In the extreme western of Santa Catarina is the most important poisonous plant that affects cattle. This study reports the occurrence of the spontaneous poisoning by Cestrum intermedium in cattle, in the municipality of Jupiá, western Santa Catarina State. The clinical signs were characterized by aggressiveness, incoordination, fasciculation, esternal decubitus, developing to lateral decubitus followed by paddling and death. The main gross finding was observed in the liver, and included pronounced lobular pattern. Were observed in the histopathology, hepatocellular necrosis ranging from centrilobular to diffuse and congestion associated with hepatocyte vacuolization in the portal zones from areas of centrolobular necrosis. No additional known hepatotoxic plant was found in the paddock where the cattle had been kept.

Key words: Cestrum intermedium, poisoning, cattle.

${ }^{1}$ Laboratório de Patologia Animal, Centro de Ciências Agroveterinárias (CAV), Universidade do Estado de Santa Catarina (UDESC), Lages, SC/ Brasil. ${ }^{2}$ Companhia Integrada de Desenvolvimento Agrícola de Santa Catarina - CIDASC, SC. CORRESPONDÊNCIA: F.H. Furlan [fhfurlan@gmail.com]. 


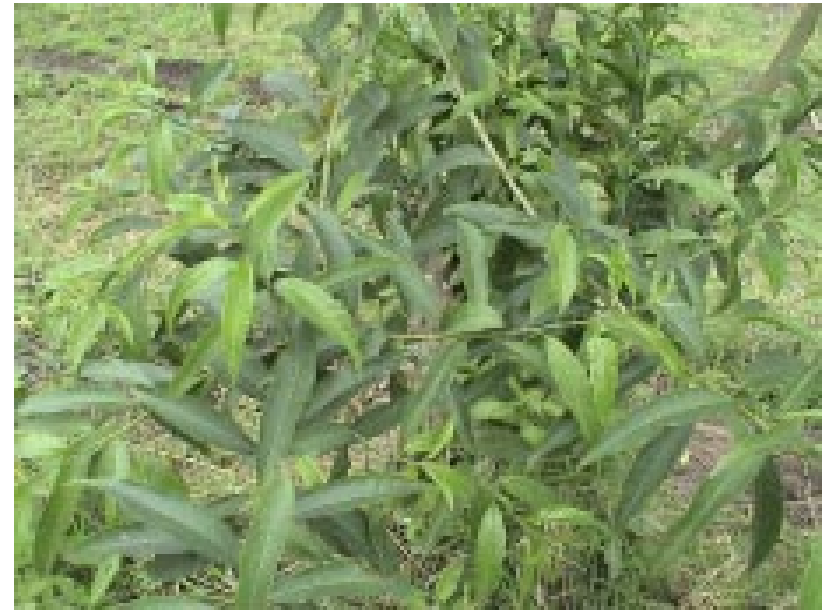

Figura 1. Cestrum intermedium

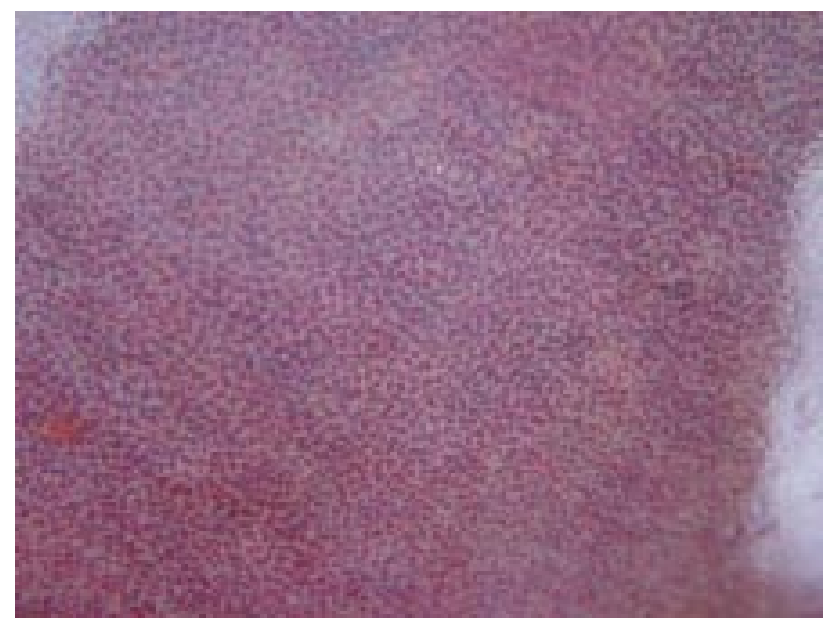

Figura 2. Superfície capsular do fígado mostrando aspecto de noz moscada, intoxicação espontânea por C. intermedium, bovino 3 .

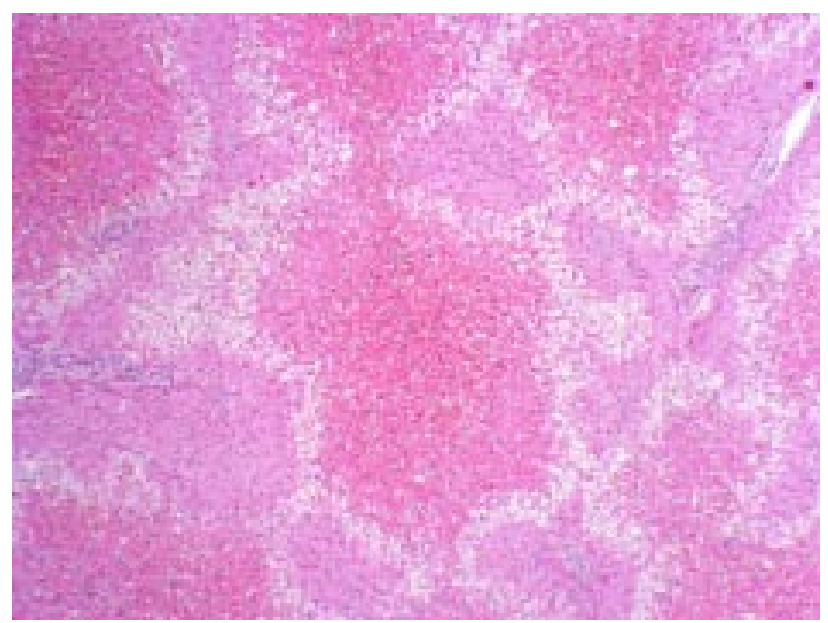

Figura 3. Necrose e hemorragia centrolobular acompanhada de degeneração vacuolar nas áreas adjacentes. Bovino 2. Intoxicação espontânea por $C$. intemedium

\section{INTRODUÇÃO}

Cestrum intermedium é um arbusto da família Solanaceae, encontrado em capoeiras e terrenos baldios, medindo até $5 \mathrm{~m}$ de altura. Ocorre principalmente no oeste de Santa Catarina, sudoeste do Paraná e noroeste do Rio Grande do Sul; nessas regiões, é conhecido pelos nomes populares de coerana e mata-boi [6-7]. No extremo oeste de Santa Catarina é a planta tóxica de maior importância para bovinos [6]. Clinicamente, bovinos intoxicados por essa planta apresentam perda de apetite, atonia ruminal, andar lento e com membros afastados, fezes secas com muco e estrias de sangue, tremores musculares, decúbito, às vezes manifestações de agressividade, movimentos de pedalagem e morte. Na necropsia, as principais lesões são verificadas no fígado, que apresenta coloração alaranjada e aspecto de noz-moscada, com edema na parede da vesícula biliar. Microscopicamente observa-se no fígado necrose de coagulação centro-lobular, às vezes com degeneração vacuolar nas áreas adjacentes [6]. O objetivo deste trabalho é relatar um surto de intoxicação espontânea por Cestrum intermedium em bovinos, no Estado de Santa Catarina.

\section{RELATO DE CASO}

A doença ocorreu em uma propriedade no município de Jupiá, extremo Oeste de Santa Catarina. O histórico e sinais clínicos foram obtidos junto ao proprietário e veterinários dos animais. Os 193 bovinos eram criados em regime extensivo e foram mantidos, por dois meses, em piquetes com grande quantidade de Cestrum intermedium (Figura 1) com sinais de consumo. Treze animais adoeceram e morreram. A doença teve curso clínico de dois dias. Os bovinos doentes apresentavam agressividade, incoordenação, tremores musculares, decúbito esternal, evoluindo para decúbito lateral, seguido de movimentos de pedalagem e morte. Na necropsia de três bovinos ( $n^{\circ} 1,2$ e 3 ) verificou-se fígado com padrão lobular acentuado (Figura 2), caracterizado como pontos avermelhados circundados por linhas claras e edema na parede da vesícula biliar que, no bovino 2 , era acompanhado de hemorragia. No cólon e reto, as fezes eram ressecadas e envolvidas por muco, nos bovinos 2 e 3 , e por estrias de sangue, no bovino 1 . $\mathrm{O}$ bovino 2 apresentava intestino delgado, com equimoses na serosa e conteúdo sanguinolento. Fragmentos de 
tecidos dos três animais foram coletados em formalina tamponada $10 \%$. As amostras foram processadas para exame histológico e coradas pelo método de Hematoxilina-Eosina (HE). Microscopicamente observou-se, em dois animais (bovinos 2 e 3), necrose e congestão centrolobular acentuadas, com degeneração vacuolar dos hepatócitos adjacentes (Figura 3). O bovino 1 apresentava necrose lobular massiva, acompanhada de hemorragia centrolobular.

\section{DISCUSSÃO E CONCLUSÃO}

Os sinais clínicos, lesões macroscópicas e histopatológicas, caracterizam o quadro clínico e lesional como hepatopatia tóxica aguda. Os agentes de ação hepatotóxica aguda, com importância econômica para bovinocultura, constituem um grupo bem representado no Brasil. Entre eles, destacam-se as plantas Cestrum intermedium [6], C. laevigatum [3], C. parqui [9], C. corymbosum [5], Dodonea sp [2], Xanthium sp [1], Vernonia molissima [4] e a lagarta Perreia flaviceps. Embora a intoxicação por $P$. flaviceps seja relatada em bovinos e ovinos apenas no Rio Grande do Sul $[8,10]$, essa lagarta também tem sido encontrada em pastagens no Estado de Santa Catarina, com relatos de morte de bovinos após o seu consumo [A. Gava, dados não publicados]. Na intoxicação por esses agentes, a principal lesão histológica é encontrada no fígado, e caracteriza-se por necrose de coagulação centrolobular à massiva associada, ou não, à degeneração vacuolar adjacente [1-6,8-10]. Portanto, baseando-se apenas pelos achados histológicos, não é possível estabelecer a etiologia da intoxicação, sendo necessário avaliar os dados epidemiológicos, constatar a presença da planta envolvida e sinais de seu consumo.

$\mathrm{Na}$ propriedade em que ocorreram as mortes dos bovinos, a doença iniciou depois que os animais foram transferidos para piquetes invadidos por $C$. Intermedium. Esta era a única planta de ação hepatotóxica aguda presente no local, e tinha sinais de intenso consumo pelos animais. A escassez de pastagem, e a grande quantidade dessa planta nos piquetes em que estavam os animais, foram os principais fatores que levaram os bovinos a consumi-la. Essa condição foi verificada também na intoxicação por Cestrum intermedium [6], C. laevigatum [3], C. parqui [9], $C$. corymbosum [5], Dodonea sp [2], Xanthium sp [1] e Vernonia molissima [4]. Não existe tratamento eficaz para intoxicação por $C$. intermedium, recomenda-se, nesses casos, a retirada dos animais dos piquetes invadidos e/ou a limpeza dos mesmos [6]. Os dados epidemiológicos, e a presença da planta com sinais de consumo nos piquetes, associados aos sinais clínicos, lesões macro e microscópicas, caracterizam a intoxicação por Cestrum intermedium em bovinos.

\section{REFERÊNCIAS}

1 Colodel E.M., Driemeier D. \& Pilati C. 2000. Intoxicação experimental pelos frutos de Xanthium cavanillesii (Asteraceae) em bovinos. Pesquisa Veterinária Brasileira. 20: 31-38.

2 Colodel E. M., Traverso S.D., Seitz A.L., Correa A.M.R., Driemeier D., Oliveira F.N. \& Gava A. 2003. Spontaneous poisoning by Dodonea viscosa (Sapindaceae) in cattle. Veterinary and Human Toxicology. 45: 147-148.

3 Döbereiner J., Tokarnia C.H. \& Canella C.F.C. 1969. Intoxicação por Cestrum laevigatum Schlecht., a causa da mortandade em bovinos no Estado do Rio de Janeiro. Pesquisa Agropecuária Brasileira. 4: 165-193.

4 Döbereiner J., Tokarnia C.H. \& Purisco E. 1976. Vernonia mollissima, planta tóxica responsável por mortandades de bovinos no sul do Mato Grosso. Pesquisa Agropecuária Brasileira. 11: 49-58.

5 Gava A., Stolf L., Pilati C., Neves D.S. \& Viganó L. 1991. Intoxicação por Cestrum corymbosum var. hirsutum (Solanaceae) em bovinos no Estado de Santa Catarina. Pesquisa Veterinária. Brasileira. 11: 71-74.

6 Gava A., Stolf L., Varaschin M. S., Neves D. S., Tigre A.P. \& Lesmann F. 1996. Intoxicação por Cestrum intermedium (Solanaceae) em Bovinos. Pesquisa Veterinária. Brasileira. 16: 117-120.

7 Lorenzi H. 2000. Plantas daninhas do Brasil: Terrestres, aquáticas, parasitas, tóxicas e medicinais. 5 ed. Nova Odessa-SP: Plantarum, 640p.

8 Raymundo D.L., Bezerra Jr. P.S., Bandara P.M., Pedroso P.M.O., Oliveira E.C., Pescador C.A \& Driemeier D. 2008. Spontaneous poisoning by larvae of Perreyia flavipes (Pergidae) in sheep. Pesquisa Veterinária Brasileira. 28 : 19-22.

9 Riet-Correa F., Schild A.L. \& Méndez M.C. 1986. Intoxicação por Cestrum parqui (Solanaceae) em bovinos no Rio Grande do Sul. Pesquisa Veterinária Brasileira. 6: 111-115. 
Furlan F. H., Lucioli J., Borelli V., Junior O. O. F., Rebelatto S. V., Gava A. \& Traverso S. D. 2008. Intoxicação por Cestrum intermedium (Solanaceae) em bovinos no Estado de Santa Catarina.

Acta Scientiae Veterinariae. 36(3): 281-284.

10 Soares M.P., Quevedo P.S. \& Schild A.L. 2008. Intoxicação por larvas de Perreia flaviceps na região sul do Rio Grande do Sul. Pesquisa Veterinária Brasileira. 28: 169-173. 